\title{
Morphological Smoothing and Extrapolation of Word Embeddings
}

\author{
Ryan Cotterell \\ Department of Computer Science \\ Johns Hopkins University, USA \\ ryan. cotterellejhu.edu
}

\author{
Hinrich Schütze \\ CIS \\ LMU Munich, Germany \\ inquiries@cis. Imu.org
}

\author{
Jason Eisner \\ Department of Computer Science \\ Johns Hopkins University, USA \\ jason@es. jhu.edu
}

\begin{abstract}
Languages with rich inflectional morphology exhibit lexical data sparsity, since the word used to express a given concept will vary with the syntactic context. For instance, each count noun in Czech has 12 forms (where English uses only singular and plural). Even in large corpora, we are unlikely to observe all inflections of a given lemma. This reduces the vocabulary coverage of methods that induce continuous representations for words from distributional corpus information. We solve this problem by exploiting existing morphological resources that can enumerate a word's component morphemes. We present a latentvariable Gaussian graphical model that allows us to extrapolate continuous representations for words not observed in the training corpus, as well as smoothing the representations provided for the observed words. The latent variables represent embeddings of morphemes, which combine to create embeddings of words. Over several languages and training sizes, our model improves the embeddings for words, when evaluated on an analogy task, skip-gram predictive accuracy, and word similarity.
\end{abstract}

\section{Introduction}

Representations of words as high-dimensional real vectors have been shown to benefit a wide variety of NLP tasks. Because of this demonstrated utility, many aspects of vector representations have been explored recently in the literature. One of the most interesting discoveries is that these representations capture meaningful morpho-syntactic and semantic properties through very simple linear relations: in a semantic vector space, we observe that

$$
v_{\text {talked }}-v_{\text {talk }} \approx v_{\text {drank }}-v_{\text {drink }}
$$

That this equation approximately holds across many morphologically related 4-tuples indicates

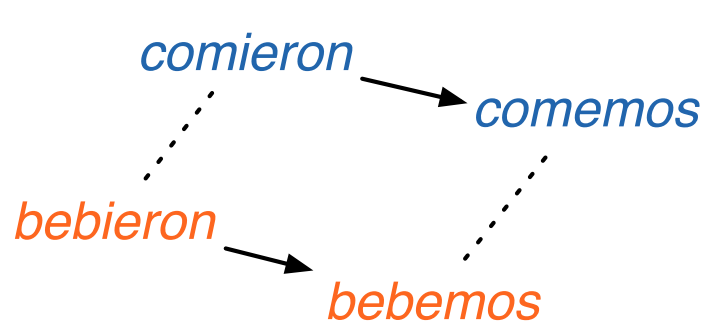

Figure 1: A visual depiction of the vector offset method for morpho-syntactic analogies in $\mathbb{R}^{2}$. We expect bebieron and bebemos to have the same relation (vector offset shown as solid vector) as comieron and comemos.

that the learned embeddings capture a feature of English morphology - adding the past tense feature roughly corresponds to adding a certain vector. Moreover, manipulating this equation yields what we will call the vector offset method (Mikolov et al., 2013c) for approximating other vectors. For instance, if we only know the vectors for the Spanish words comieron (ate), comemos (eat) and bebieron (drank), we can produce an approximation of the vector for bebemos (drink), as shown in Figure 1.

Many languages exhibit much richer morphology than English. While English nouns commonly take two forms - singular and pluralCzech nouns take 12 and Turkish nouns take over 30. This increase in word forms per lemma creates considerable data sparsity. Fortunately, for many languages there exist large morphological lexicons, or better yet, morphological tools that can analyze any word form-meaning that we have analyses (usually accurate) for forms that were unobserved or rare in our training corpus.

Our proposed method runs as a fast postprocessor (taking under a minute to process 100dimensional embeddings of a million observed word types) on the output of any existing tool that constructs word embeddings, such as WORD2VEC. 


\begin{tabular}{|c|c|c|c|c|}
\hline & \multicolumn{2}{|c|}{ Indicative } & \multicolumn{2}{|c|}{ Subjunctive } \\
\hline & $\mathrm{Sg}$ & $\mathrm{Pl}$ & $\mathrm{Sg}$ & $\mathrm{Pl}$ \\
\hline 1 & bebo & bebemos & beba & bebamos \\
\hline 2 & bebes & bebéis & bebas & bebáis \\
\hline 3 & bebe & beben & beba & beban \\
\hline
\end{tabular}

Table 1: The paradigm of the Spanish verb BEBER (to drink). The paradigm actually consists of $>40$ word forms; only the present tense portion is shown here.

In this output, some embeddings are noisy or missing, due to sparse training data. We correct these problems by using a Gaussian graphical model that jointly models the embeddings of morphologically related words. Inference under this model can smooth the noisy embeddings that were observed in the WORD2VEC output. In the limiting case of a word for which no embedding was observed (equivalent to infinite noise), inference can extrapolate one based on the observed embeddings of related words-a kind of global version of the vector offset method. The structure of our graphical model is defined using morphological lexicons, which supply analyses for each word form.

We conduct a comprehensive study of our ability to modify and generate vectors across five languages. Our model also dramatically improves performance on the morphological analogy task in many cases: e.g., accuracy at selecting the nominative plural forms of Czech nouns is $89 \%$, ten times better than the standard analogy approach.

\section{Background: Inflectional Morphology}

Many languages require every verb token to be inflected for certain properties, such as person, number, tense, and mood. A verbal paradigm such as Table 1 lists all the inflected forms of a given verb. We may refer to this verb in the abstract by its lemma, BEBER-but when using it in a sentence, we must instead select from its paradigm the word type, such as bebéis, that expresses the contextually appropriate properties. Noun tokens in a language may similarly be required to be inflected for properties such as case, gender, and number.

A content word is chosen by specifying a lemma (which selects a particular paradigm) together with some inflectional attributes (which select a particular slot within that paradigm). For example, [Lemma=EAT, Person=3, Number=Singular, Tense=PRESENT $]$ is a bundle of attribute-value pairs that would be jointly expressed in English by the word form eats (Sylak-Glassman et al., 2015).

The regularities observed by Mikolov et al. (2013c) hold between words with similar attributevalue pairs. In Spanish, the word beben "they drink" (Table 1) can be analyzed as expressing the bundle [Lemma=BEBER, Person=3, Number=PLURAL, Tense=PRESENT ]. Its vector similarity to bebemos "we drink" is due to the fact that both word forms have the same lemma BEBER. Likewise, the vector similarity of beben to comieron "they ate" is due to the conceptual similarity of their lemmas, BEBER "drink" and COMER "eat". Conversely, that beben is similar to preguntan "they ask" is caused by shared inflectional attributes [Person=3, Number $=$ PluRAL, Tense $=$ PRESENT $]$. Under cosine similarity, the most similar words are often related on both axes at once: e.g., one of the word forms closest to beben typically is comen "they eat".

\section{Approach}

Following this intuition, we fit a directed Gaussian graphical model (GGM) that simultaneously considers (i) each word's embedding (obtained from an embedding model like WORD2VEC) and (ii) its morphological analysis (obtained from a lexical resource). We then use this model to smooth the provided embeddings, and to generate embeddings for unseen inflections. For a lemma covered by the resource, the GGM can produce embeddings for all its forms (if at least one of these forms has a known embedding); this can be extended to words not covered using a guesser like MORFESSOR (Creutz and Lagus, 2007) or CHIPMunk (Cotterell et al., 2015a).

A major difference of our approach from related techniques is that our model uses existing morphological resources (e.g., morphological lexicons or finite-state analyzers) rather than semantic resources (e.g., WordNet (Miller et al., 1990) and PPDB (Ganitkevitch et al., 2013)). The former tend to be larger: we often can analyze more words than we have semantic representations for.

It would be possible to integrate our GGM into the training procedure for a word embedding system, making that system sensitive to morphological attributes. However, the postprocessing approach in our present paper lets us use any existing word embedding system as a black box. It is simple to implement, and turns out to get excellent results, which will presumably improve further as 


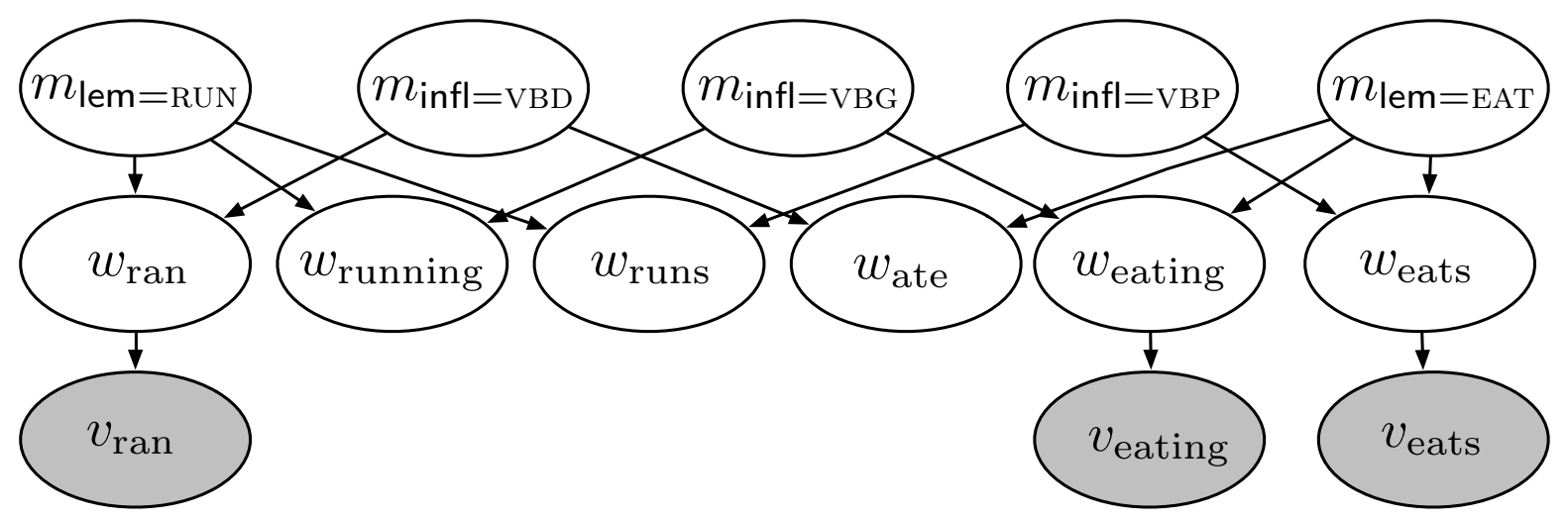

Figure 2: A depiction of our directed Gaussian graphical model (GGM) for the English verbal paradigm. Each variable represents a vector in $\mathbb{R}^{n}$; thus, this is not the traditional presentation of a GGM in which each node would be a single realvalued random variable, but each node represents a real-valued random vector. The shaded nodes $v_{i}$ at the bottom are observed word embeddings. The nodes $w_{i}$ at the middle layer are smoothed or extrapolated word embeddings. The nodes $m_{k}$ at the top are latent embeddings of morphemes.

better black boxes become available.

\section{A Generative Model}

Figure 2 draws our GGM's structure as a Bayes net. In this paper, we loosely use the term "morpheme" to refer to an attribute-value pair (possibly of the form Lemma=... ). Let $\mathcal{M}$ be the set of all morphemes. In our model, each morpheme $k \in \mathcal{M}$ has its own latent embedding $m_{k} \in \mathbb{R}^{n}$. These random variables are shown as the top layer of Figure 2. We impose an IID spherical Gaussian prior on them (similar to $\mathrm{L}_{2}$ regularization with strength $\lambda>0$ ):

$$
m_{k} \sim \mathcal{N}\left(\mathbf{0}, \lambda^{-1} I\right), \forall k
$$

Let $\mathcal{L}$ be the lexicon of all word types that appear in our lexical resource. (The noun and verb senses of bat are separate entries in $\mathcal{L}$.) In our model, each word $i \in \mathcal{L}$ has a latent embedding $w_{i} \in \mathbb{R}^{n}$. These random variables are shown as the middle layer of Figure 2. We assume that each $w_{i}$ is simply a sum of the $m_{k}$ for its component morphemes $M_{i} \subseteq \mathcal{M}$ (shown in Figure 2 as $w_{i}$ 's parents), plus a Gaussian perturbation:

$$
w_{i} \sim \mathcal{N}\left(\sum_{k \in M_{i}} m_{k}, \Sigma_{i}\right), \forall i
$$

This perturbation models idiosyncratic usage of word $i$ that is not predictable from its morphemes. The covariance matrix $\Sigma_{i}$ is shared for all words $i$ with the same coarse POS (e.g., VERB).

Our system's output will be a guess of all of the $w_{i}$. Our system's input consists of noisy estimates $v_{i}$ for some of the $w_{i}$, as provided by a black-box word embedding system run on some large corpus $\mathcal{C}$. (Current systems estimate the same vector for both senses of bat.) These observed random variables are shown as the bottom layer of Figure 2 . We assume that the black-box system would have recovered the "true" $w_{i}$ if given enough data, but instead it gives a noisy small-sample estimate

$$
v_{i} \sim \mathcal{N}\left(w_{i}, \frac{1}{n_{i}} \Sigma_{i}^{\prime}\right), \forall i
$$

where $n_{i}$ is the count of word $i$ in training corpus $\mathcal{C}$.

This formula is inspired by the central limit theorem, which guarantees that $v_{i}$ 's distribution would approach (4) (as $n_{i} \rightarrow \infty$ ) if it were estimated by averaging a set of $n_{i}$ noisy vectors drawn IID from any distribution with mean $w_{i}$ (the truth) and covariance matrix $\Sigma_{i}^{\prime}$. A system like WORD2VEC does not precisely do that, but it does choose $v_{i}$ by aggregating (if not averaging) the influences from the contexts of the $n_{i}$ tokens.

The parameters $\lambda, \Sigma_{i}, \Sigma_{i}^{\prime}$ now have likelihood

$p(\boldsymbol{v})=\int p(\boldsymbol{v}, \boldsymbol{w}, \boldsymbol{m}) d \boldsymbol{w} d \boldsymbol{m}$, where

$p(\boldsymbol{v}, \boldsymbol{w}, \boldsymbol{m})=\prod_{k \in \mathcal{M}} p\left(m_{k}\right) \cdot \prod_{i \in \mathcal{L}} p\left(w_{i} \mid m_{k}: k \in M_{i}\right)$

Here $\boldsymbol{m}=\left\{m_{k}: k \in \mathcal{M}\right\}$ represents the collection of all latent morpheme embeddings, and similarly $\boldsymbol{w}=\left\{w_{i}: i \in \mathcal{L}\right\}$ and $\boldsymbol{v}=\left\{v_{i}: i \in \mathcal{L}\right\}$. We take $p\left(v_{i} \mid w_{i}\right)=1$ if no observation $v_{i}$ exists.

How does the model behave qualitatively? If $\hat{w}_{i}$ is the MAP estimate of $w_{i}$, then $\hat{w}_{i} \rightarrow v_{i}$ as $n_{i} \rightarrow \infty$, but $\hat{w}_{i} \rightarrow \sum_{k \in M_{i}} m_{k}$ as $n_{i} \rightarrow 0$. This 
is because (3) and (4) are in tension; when $n_{i}$ is small, (4) is weaker and we get more smoothing. The morpheme embeddings $m_{k}$ are largely determined from the observed embeddings $v_{i}$ of the frequent words (since $m_{k}$ aims via (2)-(3) to explain $w_{i}$, which $\approx v_{i}$ when $i$ is frequent). That determines the compositional embedding $\sum_{k \in M_{i}} m_{k}$ toward which the $w_{i}$ of a rarer word is smoothed (away from $v_{i}$ ). If $v_{i}$ is not observed or if $n_{i}=0$, then $\hat{w}_{i}=\sum_{k \in M_{i}} m_{k}$ exactly.

\section{Inference}

Suppose first that the model parameters are known, and we want to reconstruct the latent vectors $w_{i}$. Because the joint density $p(\boldsymbol{v}, \boldsymbol{w}, \boldsymbol{m})$ in (6) is a product of (sometimes degenerate) Gaussian densities, it is itself a highly multivariate Gaussian density over all elements of all vectors. ${ }^{1}$ Thus, the posterior marginal distribution of each $w_{i}$ is Gaussian as well. A good deal is known about how to exactly compute these marginal distributions of a Gaussian graphical model (e.g., by matrix inversion) or at least their means (e.g., by belief propagation) (Koller and Friedman, 2009).

For this paper, we adopt a simpler methodMAP estimation of all latent vectors. That is, we seek the $\boldsymbol{w}, \boldsymbol{m}$ that jointly maximize (6). This is equivalent to minimizing

$$
\begin{aligned}
\sum_{k} \lambda\left\|m_{k}\right\|_{2}^{2}+ & \sum_{i}\left\|w_{i}-\sum_{k \in M_{i}} m_{k}\right\|_{\Sigma_{i}}^{2} \\
& +\sum_{i}\left\|v_{i}-w_{i}\right\|_{\Sigma_{i}^{\prime} / n_{i}}^{2},
\end{aligned}
$$

which is a simple convex optimization problem. ${ }^{2}$ We apply block coordinate descent until numerical convergence, in turn optimizing each vector $m_{k}$ or $w_{i}$ with all other vectors held fixed. This finds the global minimum (convex objective) and is extremely fast even when we have over a hundred million real variables. Specifically, we update

$$
m_{k} \leftarrow\left(\lambda I+\sum_{i \in W_{k}} \boldsymbol{\Xi}_{i}\right)^{-1} \sum_{i \in W_{k}} \boldsymbol{\Xi}_{i}\left(w_{i}-\sum_{j \in M_{i}, j \neq k} m_{j}\right),
$$

where $\checkmark \stackrel{\text { def }}{=} \Sigma^{-1}$ is the inverse covariance matrix and $W_{k} \stackrel{\text { def }}{=}\left\{i: k \in M_{i}\right\}$. This updates $m_{k}$ so

\footnotetext{
${ }^{1}$ Its inverse covariance matrix is highly sparse: its pattern of non-zeros is related to the graph structure of Figure 2. (Since the graphical model in Figure 2 is directed, the inverse covariance matrix has a sparse Cholesky decomposition that is even more directly related to the graph structure.)

${ }^{2}$ By definition, $\|\boldsymbol{x}\|_{A}^{2} \stackrel{\text { def }}{=} \boldsymbol{x}^{T} A \boldsymbol{x}$.
}

the partial derivatives of (7) with respect to the components of $m_{k}$ are 0 . In effect, this updates $m_{k}$ to a weighted average of several vectors. Morpheme $k$ participates in words $i \in W_{k}$, so its vector $m_{k}$ is updated to the average of the contributions $\left(w_{i}-\sum_{j \in M_{i}, j \neq k} m_{j}\right)$ that $m_{k}$ would ideally make to the embeddings $w_{i}$ of those words. The contribution of $w_{i}$ is "weighted" by the inverse covariance matrix $Z_{i}$. Because of prior (2), 0 is also included in the average, "weighted" by $\lambda I$.

Similarly, the update rule for $w_{i}$ is

$$
w_{i} \leftarrow\left(n_{i} \mathbf{\Xi}_{i}^{\prime}+\Xi_{i}\right)^{-1}\left(n_{i} \Xi_{i}^{\prime} v_{i}+\Xi_{i} \sum_{k \in M_{i}} m_{k}\right),
$$

which can similarly be regarded as a weighted average of the observed and compositional representations. ${ }^{3}$ See Appendix $\mathrm{C}$ for the derivations.

\section{Parameter Learning}

We wish to optimize the model parameters $\lambda, \Sigma_{i}, \Sigma_{i}^{\prime}$ by empirical Bayes. That is, we do not have a prior on these parameters, but simply do maximum likelihood estimation. A standard approach is the Expectation-Maximization or EM algorithm (Dempster et al., 1977) to locally maximize the likelihood. This alternates between reconstructing the latent vectors given the parameters (E step) and optimizing the parameters given the latent vectors (M step). In this paper, we use the Viterbi approximation to the E step, that is, MAP inference as described in section 5. Thus, our overall method is Viterbi EM.

As all conditional probabilities in the model are Gaussian, the M step has closed form. MLE estimation of a covariance matrix is a standard result-in our setting the update to $\Sigma_{i}$ takes the form:

$\Sigma_{c} \leftarrow \frac{1}{N_{c}} \sum_{i: c \in C(i)}\left(w_{i}-\sum_{k \in M_{i}} m_{k}\right)\left(w_{i}-\sum_{k \in M_{i}} m_{k}\right)^{T}$,

where $C(i)$ are $i$ 's POS tags, $N_{c}=|\{i \mid c \in C(i)\}|$ and $\Sigma_{c}$ is the matrix for the $c^{\text {th }}$ POS tag (the matrices are tied by POS). In this paper we simply fix $\Sigma_{i}^{\prime}=I$ rather than fitting it. ${ }^{4}$ Also, we tune the hyperparameter $\lambda$ on a development set, using grid search over the values $\{0.1,0.5,1.0\}$.

\footnotetext{
${ }^{3}$ If $v_{i}$ is not observed, take $n_{i}=0$. In fact it is not necessary to represent this $w_{i}$ during optimization. Simply omit $i$ from all $W_{k}$. After convergence, set $w_{i} \leftarrow \sum_{k \in M_{i}} m_{k}$.

${ }^{4}$ Note that it is not necessary to define it as $\lambda^{\prime} I$, introducing a new scale parameter $\lambda^{\prime}$, since doubling $\lambda^{\prime}$ would have the same effect on the MAP update rules as halving $\lambda$ and $\Sigma_{i}$.
} 
Viterbi EM can be regarded as block coordinate descent on the negative log-likelihood function, with $\mathrm{E}$ and $\mathrm{M}$ steps both improving this common objective along different variables. We update the parameters (M step above) after each 10 passes of updating the latent vectors (section 5's E step).

\section{Related Work}

Our postprocessing strategy is inspired by Faruqui et al. (2015), who designed a retrofitting procedure to modify pre-trained vectors such that their relations match those found in semantic lexicons. We focus on morphological resources, rather than semantic lexicons, and employ a generative model. More importantly, in addition to modifying vectors of observed words, our model can generate vectors for forms not observed in the training data.

Wieting et al. (2015) compute compositional embeddings of phrases, with their simplest method being additive (like ours) over the phrase's words. Their embeddings are tuned to fit observed phrase similarity scores from PPDB (Ganitkevitch et al., 2013), which allows them to smooth and extend PPDB just as we do to WORD2VEC output.

Using morphological resources to enhance embeddings at training time has been examined by numerous authors. Luong et al. (2013) used MoRFESSOR (Creutz and Lagus, 2007), an unsupervised morphological induction algorithm, to segment the training corpus. They then trained a recursive neural network (Goller and Kuchler, 1996; Socher, 2014) to generate compositional word embeddings. Our model is much simpler and faster to train. Their evaluation was limited to English and focused on rare English words. dos Santos and Zadrozny (2014) introduced a neural tagging architecture (Collobert et al., 2011) with a characterlevel convolutional layer. Qiu et al. (2014) and Botha and Blunsom (2014) both use MORFESSOR segmentations to augment WORD2VEC and a logbilinear (LBL) language model (Mnih and Hinton, 2007), respectively. Similar to us, they have an additive model of the semantics of morphemes, i.e., the embedding of the word form is the sum of the embeddings of its constituents. In contrast to us, however, both include the word form itself in the sum. Finally, Cotterell and Schütze (2015) jointly trained an LBL language model and a morphological tagger (Hajič, 2000) to encourage the embeddings to encode rich morphology. With the exception of (Cotterell and Schütze, 2015), all of the above methods use unsupervised methods to infuse word embeddings with morphology. Our approach is supervised in that we use a morphological lexicon, i.e., a manually built resource.

Our model is also related to other generative models of real vectors common in machine learning. The simplest of them is probabilistic principal component analysis (Roweis, 1998; Tipping and Bishop, 1999), a generative model of matrix factorization that explains a set of vectors via latent low-dimensional vectors. Probabilistic canonical correlation analysis similarly explains a set of pairs of vectors (Bach and Jordan, 2005).

Figure 2 has the same topology as our graphical model in (Cotterell et al., 2015b). In that work, the random variables were strings rather than vectors. Morphemes were combined into words by concatenating strings rather than adding vectors, and then applying a stochastic edit process (modeling phonology) rather than adding Gaussian noise.

\section{Experiments}

We perform three experiments to test the ability of our model to improve on WORD2VEC. To reiterate, our approach does not generate or analyze a word's spelling. Rather, it uses an existing morphological analysis of a word's spelling (constructed manually or by a rule-based or statistical system) as a resource to improve its embedding.

In our first experiment, we attempt to identify a corpus word that expresses a given set of morphological attributes. In our second experiment, we attempt to use a word's embedding to predict the words that appear in its context, i.e., the skip-gram objective of Mikolov et al. (2013a). Our third example attempts to use word embeddings to predict human similarity judgments.

We experiment on 5 languages: Czech, English, German, Spanish and Turkish. For each language, our corpus data consists of the full Wikipedia text. Table 5 in Appendix A reports the number of types and tokens and their ratio. The lexicons we use are characterized in Table 6: MorfFlex CZ for Czech (Hajič and Hlaváčová, 2013), CELEX for English and German (Baayen et al., 1993) and lexicons for Spanish and Turkish that were scraped from Wiktionary by Sylak-Glassman et al. (2015).

Given a finite training corpus $\mathcal{C}$ and a lexicon $\mathcal{L},{ }^{5}$ we generate embeddings $v_{i}$ for all word

\footnotetext{
${ }^{5} \mathcal{L}$ is finite in our experiments. It could be infinite (though still incomplete) if a morphological guesser were used.
} 

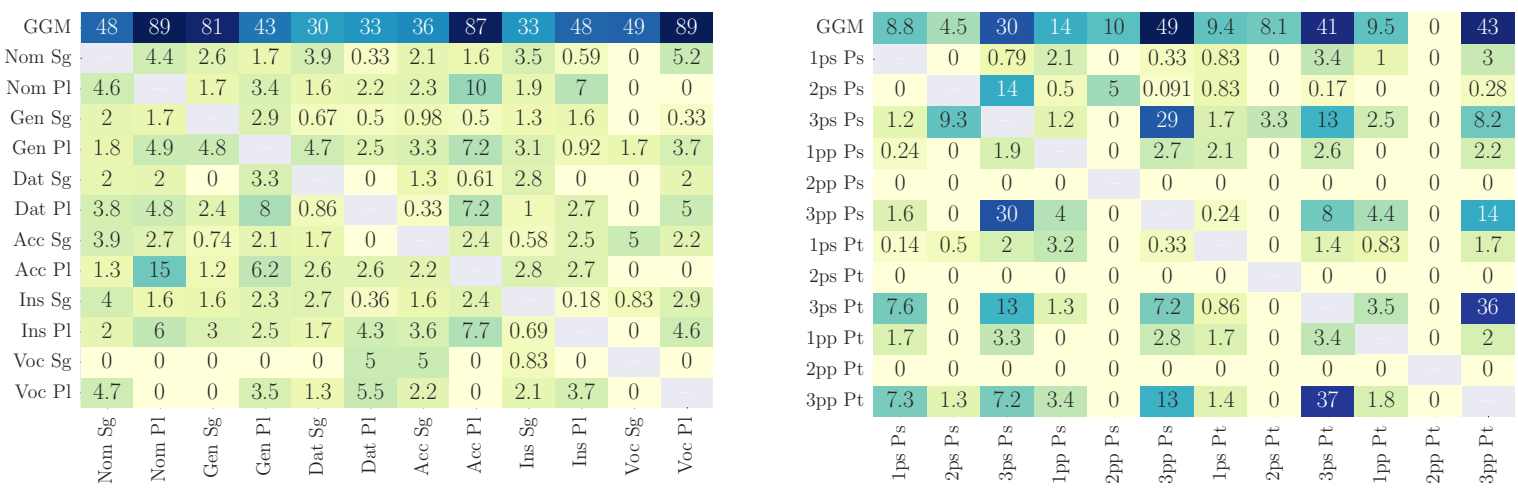

Table 2: The two tables show how the Gaussian graphical model (GGM) compares to various analogies on Czech nouns (left) and Spanish verbs (right).The numbers in each cell represent the accuracy (larger is better). The columns represent the inflection of the word $i$ to be predicted. Our GGM model is the top row. The other rows subdivide the baseline analogy results according to the inflection of source word $a$. Abbreviations: in the Czech noun table (left), the first word indicates the case and the second the number, e.g., Dat $\mathrm{Sg}=$ Dative Singular. In the Spanish verb table (right), the first word is the person and number and the second the tense, e.g., $3 \mathrm{pp} \mathrm{Pt}=3 \mathrm{rd}$-person plural past.

types $i \in \mathcal{C}$, using the GENSIM implementation (Řehưřrek and Sojka, 2010) of the WORD2VEC hierarchical softmax skip-gram model (Mikolov et al., 2013a), with a context size of 5 . We set the dimension $n$ to 100 for all experiments. ${ }^{6}$

We then apply our GGM to generate smoothed embeddings $w_{i}$ for all word types $i \in \mathcal{C} \cap \mathcal{L}$. (Recall that the noun and verb sense of bats are separate types in $\mathcal{L}$, even if conflated in $\mathcal{C}$, and get separate embeddings.) How do we handle other word types? For an out-of-vocabulary (OOV) test word $i \notin \mathcal{C}$, we will extrapolate $w_{i} \leftarrow \sum_{k \in M_{i}} m_{k}$ on demand, as the GGM predicts, provided $i \in \mathcal{L}$. If any of these morphemes $m_{k}$ were themselves never seen in $\mathcal{C}$, we back off to the mode of the prior to take $m_{k}=0 .^{7}$ Our experiments also encounter out-of-lexicon (OOL) test words $i \notin \mathcal{L}$, for which we have no morphological analysis; here we take $w_{i}=v_{i}$ (unsmoothed) if $i \in \mathcal{C}$ and $w_{i}=\mathbf{0}$ otherwise.

\subsection{Experiment 1: Extrapolation vs. Analogy}

Our first set of experiments uses embeddings for word selection. Our prediction task is to identify the unique word $i \in \mathcal{C}$ that expresses the

\footnotetext{
${ }^{6}$ An additional important hyperparameter is the number of epochs. The default value in the GENSIM package is 5, which is suitable for larger corpora. We use this value for Experiments 1 and 3. Experiment 2 involves training on smaller corpora and we found it necessary to set the number of epochs to 10 .

${ }^{7}$ One could in principle learn "backoff morphemes." For instance, if borogoves is analyzed as [ Lemma=OOV_Noun, Num=PL ], we might want $m_{\text {Lemma }=\text { OOV_Noun }} \neq \mathbf{0}$ to represent novel nouns.
}

morphological attributes $M_{i}$. To do this, we predict a target embedding $x$, and choose the most similar unsmoothed word by cosine distance, $\hat{\imath}=$ $\operatorname{argmax}_{j \in \mathcal{C}} v_{j} \cdot x$. We are scored correct if $\hat{\imath}=i$. Our experimental design ensures that $i \notin \mathcal{L}$, since if it were, we could trivially find $i$ simply by consulting $\mathcal{L}$. The task is to identify missing lexical entries, by exploiting the distributional properties in $\mathcal{C}$. ${ }^{8}$ Given the input bundle $M_{i}$, our method predicts the embedding $x=\sum_{k \in M_{i}} m_{k}$, and so looks for a word $j \in \mathcal{C}$ whose unsmoothed embedding $v_{j} \approx x$. The GGM's role here is to predict that the bundle $M_{i}$ will be realized by something like $x$.

The baseline method is the analogy method of equation (1). This predicts the embedding $x$ via the vector-offset formula $v_{a}+\left(v_{b}-v_{c}\right)$, where $a, b, c \in \mathcal{C} \cap \mathcal{L}$ are three other words sharing $i$ 's coarse part of speech such that $M_{i}$ can be expressed as $M_{a}+\left(M_{b}-M_{c}\right) \cdot{ }^{9} \quad$ Specifically, the baseline chooses $a, b, c$ uniformly at random from all possibilities. (This is not too inefficient: given $a$, at most one choice of $(b, c)$ is possible.) Note that the baseline extrapolates from the unsmoothed embeddings of 3 other words, whereas the GGM considers all words in $\mathcal{C} \cap \mathcal{L}$ that share $i$ 's morphemes.

\footnotetext{
${ }^{8}$ The argmax selection rule does not exploit the fact that the entry is missing: it is free to incorrectly return some $\hat{\imath} \in$ $\mathcal{L}$.

${ }^{9}$ More formally, $\overline{M_{i}}=\overline{M_{a}}+\left(\overline{M_{b}}-\overline{M_{c}}\right)$, if we define $\bar{M}$ by $(\bar{M})_{k}=\mathbb{I}(k \in M)$ for all morphemes $k \in \mathcal{M}$. This converts morpheme bundle $M$ to a $\{0,1\}$ indicator vector $\bar{M}$ over $\mathcal{M}$.
} 


\begin{tabular}{|c|c|c|c|c|c|c|c|c|c|c|c|c|c|}
\hline & 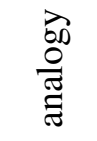 & $\sum_{0}$ & & 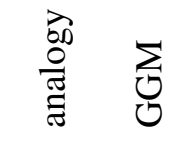 & & 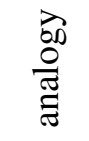 & $\sum_{0}$ & & 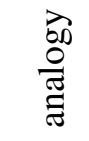 & $\sum_{0}$ & & 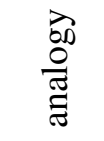 & $\sum_{0}$ \\
\hline $\operatorname{Ins}^{P}$ & .067 & $.343^{\star}$ & $\mathrm{Acc}^{S}$ & $.131 .582^{\star}$ & VBD & .052 & $.202^{\star}$ & Masc $^{S}$ Part & .178 & $.340^{\star}$ & $\operatorname{Loc}^{S}$ & .002 & $.036^{\star}$ \\
\hline $\mathrm{Gen}^{S}$ & .059 & $.632^{\star}$ & $\operatorname{Nom}^{S}$ & $.136 .571^{\star}$ & VBC & .06 & $.102^{\star}$ & Fem $^{P}$ Part & .230 & .286 & $\mathrm{Abl}^{S}$ & .001 & $.019^{\star}$ \\
\hline $\mathrm{Gen}^{P}$ & .078 & $.242^{\star}$ & $\mathrm{Dat}^{P}$ & $.074 .447^{\star}$ & $\mathrm{NN}$ & .051 & $.080^{\star}$ & Fem $^{S}$ Part & .242 & .235 & $\mathrm{Dat}^{S}$ & .001 & $.037^{\star}$ \\
\hline $\operatorname{Nom}^{P}$ & .076 & $.764^{\star}$ & $\operatorname{Acc}^{P}$ & $.075 .682^{\star}$ & VBN & .052 & $.218^{\star}$ & $\operatorname{Adj}^{S}$ & .201 & $.286^{\star}$ & $\operatorname{Acc}^{S}$ & .001 & $.023^{\star}$ \\
\hline $\operatorname{Nom}^{S}$ & .066 & $.290^{\star}$ & $\operatorname{Gen}^{P}$ & $.075 .666^{\star}$ & NNS & .052 & $.114^{\star}$ & Gerund $^{S}$ & .186 & $.449^{\star}$ & $\operatorname{Ins}^{S}$ & .001 & .004 \\
\hline $\operatorname{Voc}^{P}$ & .063 & $.789^{\star}$ & $\operatorname{Nom}^{P}$ & $.064 .665^{\star}$ & VB & .056 & $.275^{\star}$ & Gerund $^{P}$ & .172 & $.311^{\star}$ & $\operatorname{Nom}^{S}$ & .001 & $.023^{\star}$ \\
\hline & $\begin{array}{l}\text { Czech } \\
\text { houns }\end{array}$ & & & $\begin{array}{l}\text { ferman } \\
\text { nouns }\end{array}$ & & Englisl & & $\begin{array}{r}\mathrm{Sp}_{\mathrm{f}} \\
\text { ouns, } \mathrm{v}\end{array}$ & nish & & & $\begin{array}{l}\text { urkish } \\
\text { nouns }\end{array}$ & \\
\hline
\end{tabular}

Table 3: Test results for Experiment 1. The rows indicate the inflection of the test word $i$ to be predicted (superscript ${ }^{P}$ indicates plural, superscript ${ }^{S}$ singular). The columns indicate the prediction method. Each number is an average over 10 training-test splits. Improvements marked with $\mathrm{a} \star$ are statistically significant $(p<0.05)$ under a paired permutation test over these 10 runs.

Experimental Setup: A lexical resource consists of pairs (word form $i$, analysis $M_{i}$ ). For each language, we take a random $80 \%$ of these pairs to serve as the training lexicon $\mathcal{L}$ that is seen by the GGM. The remaining pairs are used to construct our prediction problems (given $M_{i}$, predict $i$ ), with a random $10 \%$ each as dev and test examples. We compare our method against the baseline method on ten such random training-test splits. We are releasing all splits for future research.

For some dev and test examples, the baseline method has no choice of the triple $a, b, c$. Rather than score these examples as incorrect, our baseline results do not consider them at all (which inflates performance). For each remaining example, to reduce variance, the baseline method reports the average performance on up to $100 a, b, c$ triples sampled uniformly without replacement.

The automatically created analogy problems $(a, b, c \rightarrow i)$ solved by the baseline are similar to those of Mikolov et al. (2013c). However, most previous analogy evaluation sets evaluate only on 4-tuples of frequent words (Nicolai et al., 2015), to escape the need for smoothing, while ours also include infrequent words. Previous evaluation sets also tend to be translations of the original English datasets-leaving them impoverished as they therefore only test morpho-syntactic properties found in English. E.g., the German analogy problems of Köper et al. (2015) do not explore the four cases and two numbers in the German adjectival system. Thus our baseline analogy results are useful as a more comprehensive study of the vector offset method for randomly sampled words.

Results: Overall results for 5 languages are shown in Table 3. Additional rows break down performance by the inflection of the target word $i$. (The inflections shown are the ones for which the baseline method is most accurate.)

For almost all target inflections, GGM is significantly better than the analogy baseline. An extreme case is the vocative plural in Czech, for which GGM predicts vectors better by more than $70 \%$. In other cases, the margin is slimmer; but GGM loses only on predicting the Spanish feminine singular participle. For Czech, German, English and Spanish the results are clear-GGM yields better predictions. This is not surprising as our method incorporates information from multiple morphologically related forms.

More detailed results for two languages are given in Table 2. Here, each row constrains the source word $a$ to have a certain inflectional tag; again we average over up to 100 analogies, now chosen under this constraint, and again we discard a test example $i$ from the test set if no such analogy exists. The GGM row considers all test examples.

Past work on morphosyntactic analogies has generally constrained $a$ to be the unmarked (lemma) form (Nicolai et al., 2015). However, we observe that it is easier to predict one word form from another starting from a form that is "closer" in morphological space. For instance, it is easier to predict Czech forms inflected in the genitive plural from forms in nominative plural, rather than the nominative singular. Likewise, it is easier to predict a singular form from another singular form rather than from a plural form. It also is easier to predict partially syncretic forms, i.e., two inflected forms that share the same orthographic string; e.g., in Czech the nominative plural and the accusative plural are identical for inanimate nouns. 

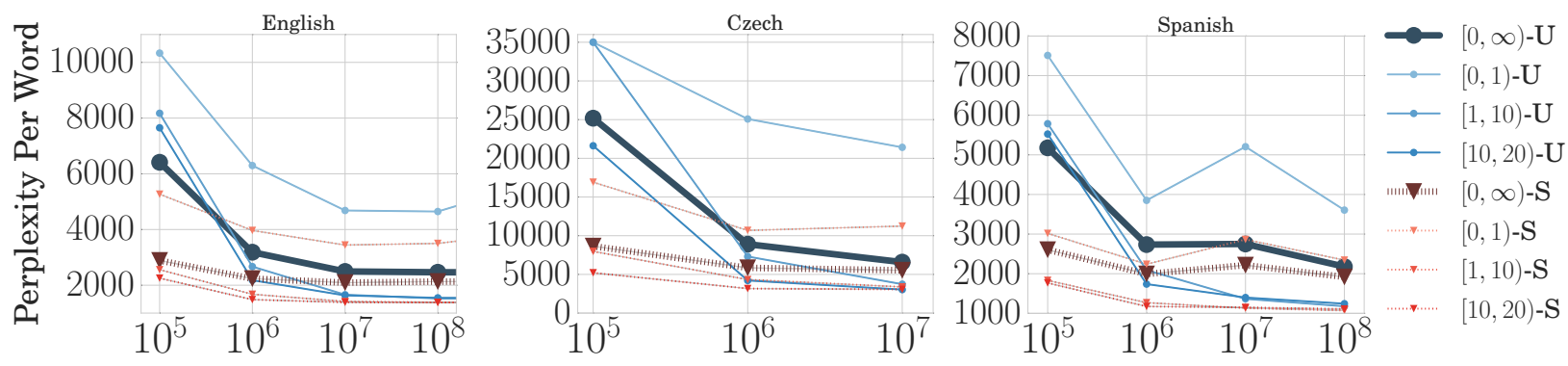

Figure 3: Results for the WORD2VEC skip-gram objective score (perplexity per predicted context word) on a held-out test corpus. The $x$-axis measures the size in tokens of the training corpus used to generate the model. We plot the held-out perplexity for the skip-gram model with Unsmoothed observed vectors $\boldsymbol{v}$ (solid $\boldsymbol{O}$ ) and Smoothed vectors $\boldsymbol{w}$ (barred $\boldsymbol{\nabla}$ ). The thickest, darkest curves show aggregate performance. The thinner, lighter versions show a breakdown according to whether the predicting word's frequency in the smallest training corpus falls in the range $[0,1),[1,10)$, or $[10,20)$ (from lightest to darkest and roughly from top to bottom). (These are the words whose representations we smooth; footnote 10 explains why we do not smooth the predicted context word.) We do not show $[20, \infty)$ since WORD2VEC randomly removes some tokens of high-frequency words ("subsampling"), similar in spirit to removing stop words. See Appendix B for more graphs.

\subsection{Experiment 2: Held-Out Evaluation}

We now evaluate the smoothed and extrapolated representations $w_{i}$. Fundamentally, we want to know if our approach improves the embeddings of the entire vocabulary, as if we had seen more evidence. But we cannot simply compare our smoothed vectors to "gold" vectors trained on much more data, since two different runs of WORD2VEC will produce incomparable embedding schemes. We must ask whether our embeddings improve results on a downstream task.

To avoid choosing a downstream task with a narrow application, we evaluate our embedding using the WORD2VEC skip-gram objective on held-out data-as one would evaluate a language model. If we believe that a better score on the WORD2VEC objective indicates generally more useful embeddings-which indeed we do as we optimize for it - then improving this score indicates that our smoothed vectors are superior. Concretely, the objective is

$$
\sum_{s} \sum_{t} \sum_{j \in[t-5, t+5], j \neq t} \log _{2} p_{\text {word2vec }}\left(T_{s j} \mid T_{s t}\right)
$$

where $T_{s}$ is the $s^{\text {th }}$ sentence in the test corpus, $t$ indexes its tokens, and $j$ indexes tokens near $t$. The probability model $p_{\text {word2vec }}$ is defined in Eq. (3) of (Mikolov et al., 2013b). It relies on an embedding of the word form $T_{s t} .{ }^{10}$ Our baseline approach

\footnotetext{
${ }^{10}$ In the hierarchical softmax version, it also relies on a separate embedding for a variable-length bit-string encoding of the context word $T_{s j}$. Unfortunately, we do not currently know of a way to smooth these bit-string encodings (also found by WORD2VEC). However, it might be possible to directly incorporate morphology into the construction of the vocabulary tree that defines the bit-strings.
}

simply uses WORD2VEC's embeddings (or $\mathbf{0}$ for OOV words $T_{s t} \notin \mathcal{C}$ ). Our GGM approach substitutes "better" embeddings when $T_{s t}$ appears in the lexicon $\mathcal{L}$ (if $T_{s t}$ is ambiguous, we use the mean $w_{i}$ vector from all $i \in \mathcal{L}$ with spelling $T_{s t}$ ).

Note that (8) is itself a kind of task of predicting words in context, resembling language modeling or a "cloze" task. Also, Taddy (2015) showed how to use this objective for document classification.

Experimental Setup: We evaluate GGM on the same 5 languages, but now hold out part of the corpus instead of part of the lexicon. We take the training corpus $\mathcal{C}$ to be the initial portion of Wikipedia of size $10^{5}, 10^{6}, 10^{7}$ or $10^{8}$. (We skip the $10^{8}$ case for the smaller datasets: Czech and Turkish). The $10^{7}$ tokens after that are the dev corpus; the next $10^{7}$ tokens are the test corpus.

Results: We report results on three languages in Figure 3 and all languages in Appendix B. Smoothing from $v_{i}$ to $w_{i}$ helps a lot, reducing perplexity by up to $48 \%$ (Czech) with $10^{5}$ training tokens and up to $10 \%$ (Spanish) even with $10^{8}$ training tokens. This roughly halves the perplexity, which in the case of $10^{5}$ training tokens, is equivalent to $8 \times$ more training data. This is a clear win for lower-resource languages. We get larger gains from smoothing the rarer predicting words, but even words with frequency $\geq 10^{-4}$ benefit. (The exception is Turkish, where the large gains are confined to rare predicting words.) See Appendix $\mathrm{B}$ for more analysis. 


\begin{tabular}{lccc} 
& English & German & Spanish \\
\hline \hline Forms / Lemma & 1.8 & 6.3 & 8.1 \\
\hline Skip-Gram & $\mathbf{5 8 . 9}$ & 36.2 & 37.8 \\
GGM & $\mathbf{5 8 . 9}$ & $\mathbf{3 7 . 6}$ & $\mathbf{4 0 . 3}$
\end{tabular}

Table 4: Word similarity results (correlations) using the WS353 dataset in the three languages, in which it is available. Since all the words in WS-353 are lemmata, we report the average inflected form to lemma ratio for forms appearing in the datasets.

\subsection{Experiment 3: Word Similarity}

As a third and final experiment, we consider word similarity using the WS-353 data set (Finkelstein et al., 2001), translated into Spanish (Hassan and Mihalcea, 2009) and German (Leviant, 2016). ${ }^{11}$ The datasets are composed of 353 pairs of words. Multiple native speakers were then asked to give an integral value between 1 and 10 indicating the similarity of that pair, and those values were then averaged. In each case, we train the GGM on the whole Wikipedia corpus for the language. Since in each language every word in the WS-353 set is in fact a lemma, we use the latent embedding our GGM learns in the experiment. In Spanish, for example, we use the learned latent morpheme embedding for the lemma BEBER (recall this takes information from every element in the paradigm, e.g., bebemos and beben), rather than the embedding for the infinitival form beber. In highly inflected languages we expect this to improve performance, because to get the embedding of a lemma, it leverages the distributional signal from all inflected forms of that lemma, not just a single one. Note that unlike previous retrofitting approaches, we do not introduce new semantic information into the model, but rather simply allow the model to better exploit the distributional properties already in the text, by considering words with related lemmata together. In essence, our approach embeds a lemma as the average of all words containing that lemma, after "correcting" those forms by subtracting off their other morphemes (e.g., inflectional affixes).

Results: As is standard in the literature, we report Spearman's correlation cofficient $\rho$ between the averaged human scores and the cosine distance between the embeddings. We report results in Table 4 . We additionally report the average num-

\footnotetext{
${ }^{11}$ This dataset has yet to be translated into Czech or Turkish, nor are there any comparable resources in these languages.
}

ber of forms per lemma. We find that we improve performance on the Spanish and German datasets over the original skip-gram vectors, but only equal the performance on English. This is not surprising as German and Spanish have roughly 3 and 4 times more forms per lemma than English. We speculate that cross-linguistically the GGM will improve word similarity scores more for languages with richer morphology.

\section{Conclusion and Future Work}

For morphologically rich languages, we generally will not observe, even in a large corpus, a high proportion of the word forms that exist in lexical resources. We have presented a Gaussian graphical model that exploits lexical relations documented in existing morphological resources to smooth vectors for observed words and extrapolate vectors for new words. We show that our method achieves large improvements over strong baselines for the tasks of morpho-syntactic analogies and predicting words in context. Future work will consider the role of derivational morphology in embeddings as well as noncompositional cases of inflectional morphology.

\section{Acknowledgments}

The first author was funded by a DAAD LongTerm Research Grant. This work was also partially supported by Deutsche Forschungsgemeinschaft (grat SCHU-2246/2-2 WordGraph) and by the U.S. National Science Foundation under Grant No. 1423276.

\section{References}

R Harald Baayen, Richard Piepenbrock, and Rijn van H. 1993. The CELEX lexical data base on CDROM.

Francis R Bach and Michael I Jordan. 2005. A probabilistic interpretation of canonical correlation analysis. Technical report, UC Berkeley.

Jan A. Botha and Phil Blunsom. 2014. Compositional Morphology for Word Representations and Language Modelling. In ICML.

Ronan Collobert, Jason Weston, Léon Bottou, Michael Karlen, Koray Kavukcuoglu, and Pavel Kuksa. 2011. Natural language processing (almost) from scratch. JMLR, 12.

Ryan Cotterell and Hinrich Schütze. 2015. Morphological word embeddings. In NAACL. 
Ryan Cotterell, Thomas Müller, Alexander Fraser, and Hinrich Schütze. 2015a. Labeled morphological segmentation with semi-Markov models. In CoNLL.

Ryan Cotterell, Nanyun Peng, and Jason Eisner. 2015b. Modeling word forms using latent underlying morphs and phonology. TACL, 3 .

Mathias Creutz and Krista Lagus. 2007. Unsupervised models for morpheme segmentation and morphology learning. ACM Transactions on Speech and Language Processing, 4(1):3.

Arthur P Dempster, Nan M Laird, and Donald B Rubin. 1977. Maximum likelihood from incomplete data via the EM algorithm. Journal of the Royal Statistical Society B, pages 1-38.

Cicero Nogueira dos Santos and Bianca Zadrozny. 2014. Learning character-level representations for part-of-speech tagging. In $I C M L$.

Manaal Faruqui, Jesse Dodge, Sujay K Jauhar, Chris Dyer, Eduard Hovy, and Noah A Smith. 2015. Retrofitting word vectors to semantic lexicons. In NAACL.

Lev Finkelstein, Evgeniy Gabrilovich, Yossi Matias, Ehud Rivlin, Zach Solan, Gadi Wolfman, and Eytan Ruppin. 2001. Placing search in context: The concept revisited. In $W W W$. ACM.

Juri Ganitkevitch, Benjamin Van Durme, and Chris Callison-Burch. 2013. PPDB: The paraphrase database. In $N A A C L$.

Christoph Goller and Andreas Kuchler. 1996. Learning task-dependent distributed representations by backpropagation through structure. Neural Networks.

Jan Hajič and Jaroslava Hlaváčová. 2013. MorfFlex CZ. LINDAT/CLARIN digital library at Institute of Formal and Applied Linguistics, Charles University in Prague.

Jan Hajič. 2000. Morphological tagging: Data vs. dictionaries. In NAACL.

Samer Hassan and Rada Mihalcea. 2009. Crosslingual semantic relatedness using encyclopedic knowledge. In EMNLP.

Daphne Koller and Nir Friedman. 2009. Probabilistic Graphical Models: Principles and Techniques. MIT press.

Maximilian Köper, Christian Scheible, and Sabine Schulte im Walde. 2015. Multilingual reliability and semantic structure of continuous word spaces. In IWCS.

Ira Leviant. 2016. Separated by an Un-common Language: Towards Judgment Language Informed Vector Space Modeling. Ph.D. thesis, Technion.
Minh-Thang Luong, Richard Socher, and C Manning. 2013. Better word representations with recursive neural networks for morphology. In CoNLL.

Tomas Mikolov, Kai Chen, Greg Corrado, and Jeffrey Dean. 2013a. Efficient estimation of word representations in vector space. In ICLR Workshop.

Tomas Mikolov, Ilya Sutskever, Kai Chen, Greg Corrado, and Jeffrey Dean. 2013b. Distributed representations of words and phrases and their compositionality. In NIPS.

Tomas Mikolov, Wen-tau Yih, and Geoffrey Zweig. 2013c. Linguistic regularities in continuous space word representations. In NAACL.

George A. Miller, Richard Beckwith, Christiane Fellbaum, Derek Gross, and Katherine J Miller. 1990. Introduction to WordNet: An on-line lexical database. International Journal of Lexicography, 3(4):235-244.

Andriy Mnih and Geoffrey Hinton. 2007. Three new graphical models for statistical language modelling. In $I C M L$.

Garrett Nicolai, Colin Cherry, and Grzegorz Kondrak. 2015. Morpho-syntactic regularities in continuous word representations: A multilingual study. In Proceedings of Workshop on Vector Space Modeling for NLP, pages 129-134.

Siyu Qiu, Qing Cui, Jiang Bian, Bin Gao, and Liu TieYan. 2014. Co-learning of word representations and morpheme representations. In COLING.

Radim Řehůřek and Petr Sojka. 2010. Software Framework for Topic Modelling with Large Corpora. In Proceedings of the LREC 2010 Workshop on New Challenges for NLP Frameworks, pages 4550 .

Sam Roweis. 1998. EM algorithms for PCA and SPCA. In NIPS

Richard Socher. 2014. Recursive Deep Learning for Natural Language Processing and Computer Vision. Ph.D. thesis, Stanford University.

John Sylak-Glassman, Christo Kirov, David Yarowsky, and Roger Que. 2015. A language-independent feature schema for inflectional morphology. In $A C L$.

Matt Taddy. 2015. Document classification by inversion of distributed language representations. In $A C L$.

Michael E Tipping and Christopher M Bishop. 1999. Probabilistic principal component analysis. Journal of the Royal Statistical Society B, 61(3):611-622.

John Wieting, Mohit Bansal, Kevin Gimpel, Karen Livescu, and Dan Roth. 2015. From paraphrase database to compositional paraphrase model and back. TACL. 